\title{
ORIENTED 6-DIMENSIONAL SUBMANIFOLDS IN THE OCTONIANS, III HIDEYA HASHIMOTO
}

Nippon Institute of Technology

Miyashiro, Saitama 345

Japan

(Received May 11, 1993 and in revised form September 27, 1993)

\begin{abstract}
In this paper, we classify 6-dimensional almost Hermitian submanifolds in the octonians $\mathbb{O}$ according to the classification introduced by A. Gray and L. Hervella. We give new examples of quasi-Kähler and $*$-Einstein submanifolds in $\mathbb{O}$. Also, we prove that a 6 -dimensional weakly $*$-Einstein Hermitian submanifold in $\mathbb{O}$ is totally geodesic.
\end{abstract}

KEY WORDS AND PHRASES. Octonians, 6-dimensional almost Hermitian submanifolds. 1991 AMS SUBJECT CLASSIFICATION CODES. 53C40, 53C15.

\section{INTRODUCTION.}

Let $\left(M^{6}, \iota\right)$ be an oriented 6-dimensional submanifold in the 8-dimensional Euclidean space $\mathbb{R}^{8}$. Throughout this paper, we shall identify $\mathbb{R}^{8}$ with the octonians $\mathbb{O}$ (or Cayley algebra) in the natural way. Taking account of the algebraic properties of the octonians, we may observe that $M^{6}$ has an almost Hermitian structure. A. Gray and L. Hervella defined the sixteen classes of almost Hermitian manifolds ([6]). In $\S 3$, we classify 6-dimensional almost Hermitian submanifolds in $O$ according to the classification given by $A$. Gray and L. Hervella. Consequently, the number of different classes (in the sense of A. Gray and L. Hervella) of almost Hermitian submanifolds in $\mathbb{O}$ is six. Last of this section, we give examples of quasi-Kähler (or $(1,2)$-symplectic) submanifolds in $\mathbb{O}$ which are not nearly Kähler and normal connections are not flat. In $\S 4$, we shall investigate the weakly $*$-Einstein submanifolds in $\mathbb{O}([13])$. We see that any Einstein Kähler manifold is necessarily a weakly *-Einstein manifold. First, we give some examples of $*$-Einstein submanifolds in $\mathbb{O}$ whose $*$-scalar curvature vanish. Also we shall show that a weakly $*$-Einstein Hermitian submanifold in $\mathbb{O}$ is a totally geodesic submanifold (Theorem 4.6). This result is a slight generalization of Chern's result ([4]) for $n=4$ in our situation.

In this paper, we adopt the same notational convention as in [1], [10] and all the manifolds are assumed to be connected and of class $C^{\infty}$ unless otherwise stated.

\section{PRELIMINARIES.}

First, we shall recall the formulation of the Spinor group $\operatorname{Spin}(7)$ given by ([1], [8]). Let $S^{6}=\{u \in \operatorname{Im} \mathbb{O} \mid\langle u, u\rangle=1\}$ where $\operatorname{Im} \mathbb{O}$ is the purely imaginary octonians. Then, for any $u \in S^{6}$, we have $u=-\bar{u}$ and $u^{2}=-u \bar{u}=-\langle u, u\rangle=-1$. So, we may use $u \in S^{6}$ to define a map $J_{u}: \mathbb{O} \rightarrow \mathbb{O}$ such that $J_{u}(x)=x u$ for any $x \in \mathbb{O}$. Each $J_{u}$ is an orthogonal complex structure on $\mathbb{O}$. It is known that $\operatorname{Spin}(7)$ is isomorphic to the subgroup of $S O(8)$ generated by the set $\left\{J_{u} \mid u \in S^{6}\right\}$. Also $S p i n(7)$ is isomorphic to the group $\{g \in S O(8) \mid g(u v)=g(u) \chi(g)(v)$ for any 
$u, r \in \mathbb{O}\}$, where $\backslash$ is the map from $S O(S)$ to itself defined $b y \backslash(g)\left(c^{\prime}\right)=g\left(g^{-1}(1) u\right)$ for any $v \in \mathbb{O}$. Then we may observe that $\left.\chi\right|_{S p m(T)} \cdot S m m(T) \rightarrow S O(T)$ is a double covering map and satisfies the following equivariance; $g(u) \times g\left(c^{\circ}\right)=\chi(g)(u \times v)$ for any $g \in S_{l} m(7)$, where $\times$ is the vector cross product defined by $u \times r=(\bar{r} u-\bar{u} u) / 2$. Now, we bhall wecall the structure equations of an

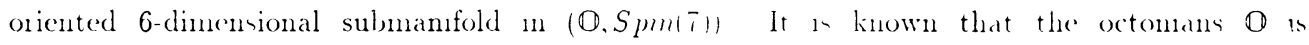

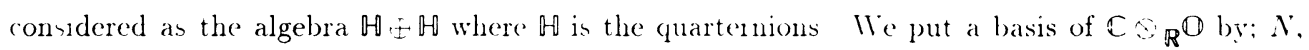
$E_{1}=\imath N, \quad E_{2}=j N, \quad E_{3}=k N, \quad \bar{N}, \quad \bar{E}_{1}=\imath \bar{N}, \quad \bar{E}_{2}=\jmath \bar{N}, \quad \bar{E}_{3}=k \bar{N}$ where $\varepsilon=(0,1) \in \mathbb{H} \oplus \mathbb{H}$. $N=(1-\sqrt{-1} \varepsilon) / 2, \bar{N}=(1+\sqrt{-1} \varepsilon) / 2 \in \mathbb{C} \odot{ }_{R} \mathbb{O}$ and $\{1, \imath, \jmath, k\}$ is the canonical basis of $\mathbb{H}$. We call this basis the standard one of $\mathbb{C} \otimes_{R} \mathbb{O}$ and a basis $(n \cdot f \cdot \bar{n}, \bar{f})$ of $\mathbb{C} \Theta_{\mathbb{R}} \mathbb{O}$ is said to be admıssible, if $(n, f, \bar{n}, \bar{f})=(N, E, \bar{N}, \bar{E}) g$ for some $g \in \operatorname{Spm}(\bar{T}) \subset M_{8 \times 8}(\mathbb{C})$. We shall identify $\operatorname{Spin}(7)$ with the admissible basis. Here, we may note that the Grassmannian manifold $G_{2}(\mathbb{O})$ of the oriented 2-planes in $\mathbb{O}$ is isomorphic to the homogeneous space $\operatorname{Spm}(7) / U(3)$. So, we can set

$$
\mathcal{F}_{,}\left(M^{6}\right)=\left\{(p ;(n, f, \bar{n}, \bar{f})) \mid-2 \sqrt{-1} n \wedge \bar{n}=T_{p}^{\perp} M^{6} \text { for any } p \in M^{6}\right\} .
$$

Then $x: F_{\iota}\left(M^{6}\right) \rightarrow M^{6}$ is a principal $U(3)$-bundle over $M^{6}$. The induced almost complex structure is defined by:

$$
\iota_{*}(J X)=\left(\iota_{*} X\right)(\eta \times \xi)
$$

for $X \in T_{p} M^{6}$, where $\xi, \quad \eta$ are an orthonormal pair of the normal vector fields and $n=\frac{1}{2}(\xi-\sqrt{-1} \eta$ ) (for details, see [1], [10]). By making use of the properties of $\operatorname{Spin}(7)$, we may observe that this almost complex structure is an invariant of $S p z n(7)$ in the following sense; let $M^{6}$ be an oriented 6 -dimensional manifold and $\iota, \iota: M^{6} \rightarrow \mathbb{O}$ be isometric immersions. If there exists $g \in \operatorname{Spin}(7)$ such that $\iota^{\prime}=g \circ \iota$ (up to parallel displacement) then $J=J^{\prime}$ where $J$ and $J^{\prime}$ are the almost complex structures on $M^{6}$ induced by the immersions $\iota$ and $\iota$, respectively. Also, we can easily see that $T^{1,0}=\operatorname{span}_{C}\left\{f_{1}, f_{2}, f_{3}\right\}$ where $T^{1,0}$ is the subbundle of the complexified tangent bundle $T M^{6} \otimes \mathbb{C}$ whose fibre is $\sqrt{-1}$-eigenspace of the almost complex structure $J$. Then we have the following structure equations:

$$
\begin{aligned}
& d \iota=f \omega+\bar{f} \bar{\omega}, \\
& d f=-n^{t} \bar{\eta}+f \kappa-\bar{n} t \bar{\theta}+\bar{f}[\theta], \\
& d n=n(\sqrt{-1} \rho)=f \eta+\bar{f} \bar{\theta}, \\
& d(\sqrt{-1} \rho)={ }^{t} \bar{\eta} \wedge \eta+{ }^{t} \theta \wedge \bar{\theta} \text {, } \\
& \left\{\begin{array}{l}
d \eta=-\eta \wedge(\sqrt{-1} \rho)-\kappa \wedge \eta-[\bar{\theta}] \wedge \bar{\theta}, \\
d \theta=-\kappa \wedge \theta+\theta \wedge(\sqrt{-1} \rho)-[\bar{\theta}] \wedge \bar{\eta},
\end{array}\right. \\
& d \kappa=\eta \wedge^{t} \bar{\eta}-\kappa \wedge \kappa+\theta \wedge^{\tau} \bar{\theta}-[\bar{\theta}] \wedge[\theta], \quad \text { (Gauss equation) }
\end{aligned}
$$

where $\rho: \mathbb{R}$-valued 1 -form, $\eta, \theta: M_{3 \times 1}(\mathbb{C})$-valued 1 -forms, and $\kappa: M_{3 \times 3}(\mathbb{C})$ valued 1 -forms on $\mathcal{F}_{\iota}\left(M^{6}\right)$ which satisfy $\kappa+{ }^{t} \bar{\kappa}=0$ and $t r \kappa+\sqrt{-1} \rho=0$. Here, $[\theta]$ is defined by 


$$
[\theta]=\left(\begin{array}{ccc}
0 & \theta^{3} & -\theta^{2} \\
-\theta^{3} & 0 & \theta^{1} \\
\theta^{2} & -\theta^{1} & 0
\end{array}\right)
$$

where $\theta={ }^{t}\left(\theta^{1}, \theta^{2}, \theta^{3}\right)$. By (2.2) and (2.3), the second fundamental form $\theta$ is given by

$$
\Pi=-2 \operatorname{Re}\left\{\left({ }^{t} \bar{b} \circ \omega+{ }^{t} \theta \circ \bar{\omega}\right) n\right\}
$$

Applying the Cartan's lemma, we may conclude that there exists $3 \times 3$ matrices of functions $A, B, C$ on $\mathcal{F}_{\imath}\left(M^{6}\right)$ (with complex values) satisfying

$$
\begin{gathered}
A={ }^{t} A, \quad C={ }^{t} C, \\
\left(\begin{array}{l}
\eta \\
\theta
\end{array}\right)=\left(\begin{array}{cc}
\bar{B} & \bar{A} \\
{ }^{B} & \bar{C}
\end{array}\right)\left(\begin{array}{l}
\omega \\
\bar{\omega}
\end{array}\right) .
\end{gathered}
$$

Hence, we have the following canonical splittings:

$$
\Pi^{2,0}=\left(-{ }^{t} \omega \circ A \omega\right) n, \Pi^{1,1}=\left(-{ }^{t} \bar{\omega} \circ{ }^{t} B \omega-{ }^{t} \omega \circ B \bar{\omega}\right) n, \Pi^{0,2}=\left(-{ }^{t} \bar{\omega} \circ \bar{C} \bar{\omega}\right) n .
$$

More explicitely, let $\left\{e_{1}, J e_{1}, e_{2}, J e_{2}, e_{3}, J e_{3}\right\}$ (resp. $\{\xi, J \xi\}$ ) be the orthonormal basis of $T_{p} M^{6}$ (resp. $T_{p}^{\perp} M^{6}$ ) at $p \in M^{6}$. Then we have

$$
\left\{\begin{array}{l}
f_{\mathfrak{t}}=\frac{1}{2}\left(e_{\mathrm{t}}-\sqrt{-1} J e_{\mathfrak{\imath}}\right) \quad \text { for } \iota=1,2,3 \\
n=\frac{1}{2}(\xi-\sqrt{-1} J \xi)
\end{array}\right.
$$

By (2.9) and (2.10), we have

$$
\begin{cases}<\Pi\left(f_{\imath}, f_{\jmath}\right), & \bar{n}>=-\frac{1}{2} A_{\imath \jmath} \\ <\Pi\left(f_{\imath}, \bar{f}_{\jmath}\right), & \bar{n}>=-\frac{1}{2} B_{\imath \jmath} . \\ <\Pi\left(\bar{f}_{\imath}, \bar{f}_{\jmath}\right), & \bar{n}>=-\frac{1}{2} \bar{C}_{\imath \jmath} .\end{cases}
$$

for any $i, j=1,2,3$.

Next, we give the representation of Ricci tensor $\rho$, scalar curvature $\tau$, Ricci $*$-tensor $\rho *$, and *-scalar curvature $\tau *$ by $A, B$ and $C$ which are defined by

$$
\begin{array}{cc}
\rho(x, y)=\sum_{i=1}^{6}<R\left(e_{\imath}, x\right) y, e_{\mathfrak{\imath}}>, & \tau=\sum_{i=1}^{6} \rho\left(e_{\imath}, e_{i}\right), \\
\rho *(x, y)=\frac{1}{2} \sum_{i=1}^{6}<R\left(e_{\imath}, J e_{\imath}\right) J y, x>, & \tau *=\sum_{i=1}^{6} \rho^{*}\left(e_{\imath}, e_{\imath}\right),
\end{array}
$$

for any $x, y \in T_{p} M^{6}, p \in M^{6}$, where $\left\{e_{\imath}\right\}$ is an orthonormal basis of $T_{p} M^{6}$. Then, we have

PROPOSITION 2.1. Let $M^{6}=\left(M^{6}, J,<,>\right)$ be a 6 -dimensional almost Hermitian submanifold in $\mathbb{O}$. Then, we have the following

(1) $\rho(x, y)=2 \operatorname{tr} B\left({ }^{t} \alpha C \beta+{ }^{t} \alpha \bar{B} \bar{\beta}+{ }^{t} \bar{\alpha} \bar{B} \beta+{ }^{t} \bar{\alpha} \bar{A} \bar{\beta}\right)$ 


$$
\begin{aligned}
& +2 \overline{\operatorname{tr} B\left({ }^{t} \alpha C \beta+{ }^{t} \alpha^{t} \bar{B} \bar{\beta}+{ }^{t} \bar{\alpha} \bar{B} \beta+{ }^{t} \bar{\alpha} \bar{A} \bar{\beta}\right)} \\
& -\left[{ }^{t} \alpha\left(A \bar{B}+B C+{ }^{t}(A \bar{B}+B C)\right) \beta+{ }^{t} \alpha\left(A \bar{A}+B^{t} \bar{B}+{ }^{t} \bar{B} B+C \bar{C}\right) \bar{\beta}\right. \\
& \left.+{ }^{t} \bar{\alpha}\left(\bar{A} A+\bar{B}^{t} B+{ }^{t} B \bar{B}+\bar{C} C\right) 3+{ }^{t} \bar{\alpha}\left(\bar{A} B+\bar{B} \bar{C}+{ }^{t}(\bar{A} B+\bar{B} \bar{C})\right) \bar{\beta}\right]
\end{aligned}
$$

(2) $\tau=4\left(4|\operatorname{tr} B|^{2}-\operatorname{tr} A \bar{A}-2 \operatorname{tr}^{t} \bar{B} B-\operatorname{tr} C \bar{C}\right)$,

(3) $\rho *(x, y)={ }^{t} \alpha\left(A \bar{B}-B C-{ }^{t}(A \bar{B}-B C)\right) \beta-{ }^{t} \alpha\left(A \bar{A}-B^{t} \bar{B}-{ }^{t} \bar{B} B+C \bar{C}\right) \bar{\beta}$

$$
-{ }^{t} \bar{\alpha}\left(\bar{A} A-\bar{B}^{t} B-{ }^{t} B \bar{B}+\bar{C} C\right) \beta+{ }^{t} \bar{\alpha}\left(\bar{A} B-\bar{B} \bar{C}-{ }^{t}(\bar{A} B-\bar{B} \bar{C})\right) \bar{\beta},
$$

(4) $\tau *=-4\left(\operatorname{tr} A \bar{A}-2 \operatorname{tr}^{t} \bar{B} B+\operatorname{tr} C \bar{C}\right)$,

where $x=f \alpha+\bar{f} \bar{\alpha}, y=f \beta+\bar{f} \bar{\beta}$.

PROOF. (1). By Gauss equation and (2.11), we get

$$
\begin{aligned}
& \rho(x, y) \\
& =<6 H, \Pi(x, y)>-2 \sum_{i=1}^{3}\left\{<\Pi\left(x, f_{\mathfrak{\imath}}\right), \Pi\left(\bar{f}_{\mathfrak{}}, y\right)>+<\Pi\left(x, \bar{f}_{\mathfrak{\imath}}\right), \Pi\left(f_{\mathfrak{t}}, y\right)>\right\} \\
& =-4<\operatorname{tr} B \cdot n+\operatorname{tr} \bar{B} \cdot \bar{n}, \Pi(f \alpha+\bar{f} \bar{\alpha}, f \beta+\bar{f} \bar{\beta})> \\
& \quad-2 \sum_{i=1}^{3}\left\{<\Pi\left(f \alpha+\bar{f} \bar{\alpha}, f_{\imath}\right), \Pi\left(\bar{f}_{\imath}, f \beta+\bar{f} \bar{\beta}\right)>\right. \\
& \left.\quad+<\Pi\left(f \alpha+\bar{f} \bar{\alpha}, \bar{f}_{\imath}\right), \Pi\left(f_{\imath}, f \beta+\bar{f} \bar{\beta}\right)>\right\},
\end{aligned}
$$

By (2.8), we get them desired equality. From (1), we have (2). (3): By (2.9), we get

$$
\begin{aligned}
\rho *(x, y) & =-2 \sqrt{-1} \sum_{i=1}^{3}\left\langle R\left(f_{\imath}, \bar{f}_{\imath}\right) J y, x>\right. \\
& =-2 \sqrt{-1} \sum_{i=1}^{3}\left\langle R\left(f_{\imath}, \bar{f}_{\imath}\right)(\sqrt{-1}(f \beta-\bar{f} \bar{\beta})), f \alpha+\bar{f} \bar{\alpha}>\right.
\end{aligned}
$$

By Guass equation, we get (3). From (3), we get (4).

3. SOME CLASSES OF $\left(\mathbf{M}^{6}, \imath\right)$.

In this section, we adopt the same notational convention as in [6]. First, we recall the following.

PROPOSITION 3.1 [1]. An almost Hermitian submanifold $M^{6}=\left(M^{6}, J, g\right)$ in 0 is a semiKähler manifold, i.e., $\delta \Omega=0$.

By Proposition 3.1, we easily see that $W_{4}=$ the class of Kähler manifolds. Hence we see that, formally, the number of different classes is eight $=2^{3}$.

PROPOSITION 3.2 [1]. Let $M^{6}=\left(M^{6}, J, g\right)$ be a 6-dimensional almost Hermitian 
submanifold in $\mathbb{O}$. The following conditions are equivalent:

(1) $M^{6}$ is a Kähler submanifold in $\mathbb{O}\left(<\frac{\overline{\overline{d e f}}}{>} \nabla J=0\right)$,

(2) $\quad M^{6}$ is an almost Kähler submanifold in $\mathbb{O}\left(<\frac{\overline{\bar{e}} \rho}{>} d \Omega=0\right)$,

(3) $M^{6}$ belongs to the class $W_{4}$,

(4) $\quad M^{6}$ is a minimal submanifold in $\mathbb{O}$ and satisfy $\mathbb{0}^{0,2}=0$.

(5) $\quad M^{6}$ is (locally) a complex hypersurface in $\mathbb{C}^{4}=\left(\mathbb{O}, J_{u}\right)$ where $u \in S^{6} \subset \operatorname{Im} \mathbb{O}$ (fixed).

PROPOSITION 3.3 [10]. Let $M^{6}=\left(M^{6}, J, g\right)$ be a 6-dimensional almost Hermitian submanifold in $\mathbb{O}$. The following conditions are equivalent:

(1) $M^{6}$ is nearly-Kähler submanifolds in $\mathbb{O}\left(<\frac{\overline{\overline{d e}}}{d}>\left(\nabla_{X} J\right) X=0\right.$ for any $\left.X \in T M^{6}\right)$.

(2) $M^{6}$ belongs to the class $W_{1}$.

(3) $\Pi^{1,1}=\left(\operatorname{tr} \Pi^{1,1}\right) I_{3}$ and $\Pi^{0,2}=0$.

(4) $\quad M^{6}$ is (locally) a complex hypersurface in $\mathbb{C}^{4}=\left(\mathbb{O}, J_{u}\right)$ or is locally isometric to a 6 dimensional round sphere in some 7-dimensional Euclidean space and the immersion is totally umbilic.

PROPOSITION 3.4 ([1], [3], [5]). Let $M^{6}=\left(M^{6}, J,<,>\right)$ be a 6-dimensional almost Hermitian submanifold in $\mathbb{O}$. The following conditions are equivalent:

(1) $M^{6}$ is a Hermitian submanifold in $\mathbb{O}$,

(2) $\Pi^{1,1}=0$,

(3) $\Pi(x, y)+\Pi(J x, J y)=0$, for any $x, y \in T_{p} M^{6}$,

(4) $J \circ A_{\xi}+A_{\xi} \circ J=0$, for any $\xi \in T_{p}^{\perp} M^{6}$,

(5) $\quad M^{6}$ belongs to the class $W_{3}$.

REMARK. There are many examples of 6-dimensional oriented Hermitian submanifolds in O which are not Kähler ([1], [3]).

PROPOSITION 3.5 ([4], [9], [10], [15]). Let $M^{6}=\left(M^{6}, J<,>\right)$ be a 6-dimensional almost Hermitian submanifold in $\mathbb{O}$. The following conditions are equivalent.

(1) $\quad M^{6}$ is a quasi-Kähler manifold $\left(<=>\left(\nabla_{X} J\right) Y+\left(\nabla_{J X} J\right) J Y=0\right)$ (or $(1,2)$-symplectic).

(2) $d \Omega^{1,2}=d \Omega^{2,1}=0$.

(3) $\Pi^{0,2}=0$.

(4) $\Pi(x, x)-\Pi(J x, J x) \pm 2 J \Pi(x, J x)=0$, for any $x \in T_{p} M^{6}$.

(5) $\quad M^{6}$ belongs to the class $W_{1} \oplus W_{2}$.

We give examples of quasi-Kähler submanifolds in $\mathbf{O}$ which are not nearly Kähler and their normal connections are not flat, in the last of this section.

Next, we shall define two classes of almost Hermitian manifolds.

DEFINITION 3.6. An almost Hermitian manifold $M^{2 n}=\left(M^{2 n}, J<,>\right)$ belongs to the 
class $\mathcal{G}_{1}=W_{1} \oplus W_{3}$ if $\left(\nabla_{X} J\right) X-\left(\nabla_{J X} J\right) J X=0$ for any $x \in \mathfrak{X}\left(M^{6}\right) . \quad M^{2 n}$ belongs to the class $\mathrm{g}_{2}=W_{2} \oplus W_{4}$ if $\left.\underset{X, Y, Z}{\mathfrak{S}}\left\{<\left(\nabla_{X} J\right) Y, Z>-<\left(\nabla_{J,} J\right) J Y, Z\right\rangle\right\}=0$ for any $X, Y, Z \in \mathfrak{X}\left(M^{6}\right)$ where $\mathbb{S}$ is the cyclic sum.

The inclusion relations are given by

$\subset \mathcal{A} \mathscr{G}$

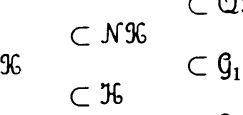

$(\subset \mathcal{A} \mathcal{H})^{\subset g_{2}}$

First, we prove the following:

PROPOSITION 3.3. Let $\left(M^{6}, \iota\right)$ be a 6 -dimensional almost Hermitian submanifold in $\mathbb{O}$. Then we have

(1) $M^{6} \in G_{1}$ if and only if $M^{6} \in \mathcal{N} \mathscr{K}$,

(2) $\quad M^{6} \in g_{2}$ if and only if $\operatorname{tr} \Pi^{1,1}=0$, i.e., $M^{6}$ is a minimal submanifold in $\mathbb{O}$.

PROOF. (1). By (2.2), we have

$$
\left(\nabla_{X} J\right) X=2 \sqrt{-1}\{\bar{f}[\theta(x)] \alpha-f[\bar{\theta}(x)] \bar{\alpha}\},
$$

for any $X=f \alpha+\bar{f} \bar{\alpha} \in \mathfrak{X}\left(M^{6}\right)$. By (3.1) and $(2.9)_{2}$, we get

$$
\left(\nabla_{X} J\right) X-\left(\nabla_{J X} J\right) J X=-4 \sqrt{-1}\left\{f[C \alpha] \bar{\alpha}-\bar{f}\left[{ }^{t} B \alpha\right] \alpha\right\}=0 .
$$

From this, we get $[C \alpha] \bar{\alpha}=0,\left[{ }^{t} B \alpha\right] \alpha=0$ for any $\alpha \in M_{3 \times 1}(\mathbb{C})$. From the definition of $[\cdot]$, we see that $C=0, B=\lambda I_{3}$. Hence we get (1).

(2) If we put, $X=f_{n}, Y=f_{\jmath}, Z=f_{k}$. Then we get

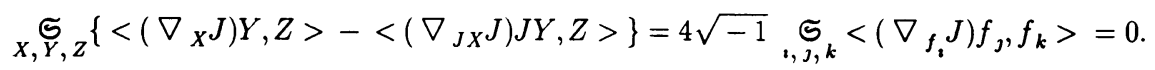

If we put, $X=\bar{f}_{\sharp}, Y=f_{\jmath}, Z=f_{k}$. Then we get the identity. From the above conditions, (2.2), (2.8) and Theorem 3.3 in [1], we have $\operatorname{tr} B=0$.

Summing up, we have

PROPOSITION 3.4. Let $\left(M^{6}, \iota\right)$ be a 6-dimensional almost Hermitian submanifold in $\mathbb{O}$. Then, $\left(M^{6}, \iota\right)$ belongs to one of six classes, $\mathscr{T}^{6}, N^{N 6}, J_{6}, Q_{96}, 996, g_{2}$ of the sixteen classes of almost Hermitian manifolds introduced by A. Gray and L. Hervella. The inclusion relations of the six classes $96, \sqrt{ } 96,36,996,996, g_{2}$ are as follows:

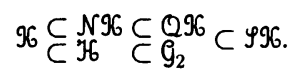

THEOREM 3.5. Let $\left(M^{6}, \iota\right)$ be an almost Hermitian submanifold in $\mathbb{O}$. If the shape operator $A_{\xi}$ commutes the almost complex structure $J$ for any normal vector $\xi$ at any point, i.e., $J \cdot A_{\xi}=A_{\xi} \cdot J$. Then we have

(1) $M^{6}$ is locally isometric to $\mathbb{R}^{6}$ and $\iota$ is totally geodesic

or

(2) $M^{6}$ is locally isometric to $S^{6}$ and $\iota$ is totally umbilic 
(3) $M^{6}$ is locally isometric to $S^{2} \times \mathbb{R}^{1}$ and, is locally of the form $\imath=\psi \times \iota d: S^{2} \times \mathbb{R}^{4} \rightarrow \mathbb{R}^{3} \times \mathbb{R}^{4}\left(\simeq \mathbb{R}^{7}\right) \subset \mathbb{O}$ where $\mathbb{R}^{3}$ is a 3 -dnuensiond Euclidean space closed under the vector cross product of $\mathbb{O}$ and $\left(S^{2}, w^{\prime}\right)$ is a 2 -dmensional phere in $\mathbb{R}^{3}$ with totally umbilical immersion $\psi$.

REMARK. Theorem 3.4 is a generalization of the results of Yano-Sumitomo [15] and A. Gray [5].

PROOF. By the assumption, we see that $M^{6}$ is a quasi-Kähler submanifold in $\mathbb{O}$. From ([10], p 84), it follows that the rank of $B$ is 3 or 1 or 0 . If the rank of $B$ is 3 , then $M^{6}$ is totally umbilic by Theorem $\mathrm{A}$ in [10]. Hence we get case (2). If the rank of $B$ is 1 , we see that the normal connection of $M^{6}$ is flat. By Theorem B in [10], we get case (3). If the rank of $B$ is 0 , by Proposition 3.4, we have $J \cdot A_{\xi}+A_{\xi} \cdot J=0$ for any $\xi \in \nu_{p}\left(M^{6}\right)$. Hence we get case (1).

We shall give examples of quasi-Kähler submanifolds in $\mathbb{O}$ whose normal connections are not flat.

EXAMPLE. Let $\iota: M^{2} \rightarrow S^{4}$ be the super-minimal, full, immersion (or real isotropic harmonic map) from the Riemann surface $M^{2}$ into the 4-dimensional sphere $S^{4}$. Let $\widetilde{p} \in S^{4} \backslash \iota\left(M^{2}\right)$ and $\pi_{\widetilde{p}}: S^{4} \backslash\{\widetilde{p}\} \rightarrow \mathbb{R}^{4}$ be the stereographic projection. We consider the following product immersion:

$$
\left(\pi_{\tilde{p}} \cdot \iota\right) \times i d: M^{2} \times \mathbb{R}^{4} \rightarrow \mathbb{H} \oplus \mathbb{H}=\mathbb{O} .
$$

Then the induced almost Hermitian structure is quasi-Kähler. In fact, we see that

$$
J\left(T_{p} M^{2}\right)=T_{p} M^{2}, J\left(T_{q} \mathbb{R}^{4}\right)=T_{q} \mathbb{R}^{4},
$$

for any $(p, q) \in M^{2} \times \mathbb{R}^{4}$. By $(3.1)$ and $i d: \mathbb{R}^{4} \rightarrow \mathbb{H}$ is totally geodesic, we may show that $\pi_{\tilde{p}} \cdot \iota$ satisfies the following condition

$$
\Pi(x, x)-\Pi(J x, J x) \pm 2 J \Pi(x, J x)=0,
$$

for any $x \in T_{p} M^{2}$. We note that the induced almost complex structure of $M^{2}$ and the almost complex structure of the normal bundle depend only on the conformal structure of the induced metrics. Also, we see that the condition (3.2) is equivalent to the ellipse of curvature of the immersion $\pi_{\tilde{p}} \cdot \iota$ being a circle. If the ellipse of curvature is a circle, then it is a conformal invariant [7]. On the other hand, since the immersion $\iota$ is a super-minimal immersion, the ellipse of curvature of the immersion $\iota$ is a circle. Also since $\pi_{\widetilde{p}}$ is a conformal mapping, we see that the immersion $\pi_{p} \cdot \iota$ satisfies the condition (3.2).

REMARK. In [2], Bryant proved that any compact Riemann surface can be immersed in $S^{4}$ as a super-minimal immersion. Hence there exist many examples. In particular, the Veronese immersion is a super-minimal immersion.

\section{4. *-EINSTEIN SUBMANIFOLD IN $\mathbb{O}$.}

In this section, we shall study weakly $*$-Einstein submanifolds in $\mathbf{O}$ and give some examples. First, we shall give the definition.

DEFINITION 4.1. An almost Hermitian manifold $\left(M^{2 n}, J,<,>\right)$ is called weakly *Einstein if $\rho *=\frac{\tau *}{2 n}<,>$ where $\tau *$ is a function on $M^{2 n}$. A weakly $*$-Einstein manifold is called a *-Einstein manifold if $\tau *$ is constant on $M^{2 n}$.

REMARK. In general, $\tau *$ is not constant. T. Koda [12] proved that $\left(\mathbb{C} P^{2} \# \overline{\mathbb{C}}^{2}, J, g\right)$ is a compact Einstein, weakly $*$-Einstein Hermitian surface whose $*$-scalar curvature is a non-constant positive function, where $g$ is the Berald-Bergery's metric. 
We shall give the equivalent conditions for weakly *-Einstein 6-dimensional submanifolds in O.

PROPOSITION 4.1. Let $\left(M^{6}, \iota\right)$ be an oriented 6-dimensional submanifold in $\mathbb{O} . \quad M^{6}$ is a weakly $*$-Einstein submanifold in $\mathbb{O}$ if and only if,

(1) ${ }^{t} \bar{B} B+B^{t} \bar{B}-A \bar{A}-C \bar{C}=\frac{\tau *}{12} I_{3}$ and $A \bar{B}-B C={ }^{t}(A \bar{B}-B C)$

or

(2) $J \cdot A_{\xi} \cdot J \cdot A_{\xi}+J \cdot A_{\eta} \cdot J \cdot A_{\eta}=-\frac{\tau *}{6} I_{6}$.

PROOF. By (3) of Proposition 2.1, we get (1). By Gauss equation, we get (2). Next, we shall give examples of $*$-Einstein submanifolds in $\mathbb{D}$.

PROPOSITION 4.2. Let $\left(M^{3}, f\right)$ and $\left(N^{3}, g\right)$ be two oriented hypersurfaces in the quaternions $\mathbb{H}$, with respective immersions $f$ and $g$, and let $f \times g: M^{3} \times N^{3} \rightarrow \mathbb{H} \oplus \mathbb{H} \simeq \mathbb{O}$ be the product immersion. Then the induced almost Hermitian structure is $*$-Einstein and its $*$-scalar curvature vanishes.

PROOF. In [9], the induced almost complex structure on $M^{3} \times N^{3}$ is given by

$$
\left\{\begin{array}{lll}
J(\xi i)=-(\eta i) \varepsilon, & J(\xi j)=-(\eta j) \varepsilon, & J(\xi k)=-(\eta k) \varepsilon \\
J((\eta i) \varepsilon)=\xi i, & J((\eta j) \varepsilon)=\xi j, & J((\eta k) \varepsilon)=\xi k
\end{array}\right.
$$

where $\xi, \eta$ are unit normal vector fields of $M^{3}, N^{3}$, respectively, and $\varepsilon=(0,1) \in \mathbb{H} \oplus \mathbb{H}$. From this, we can easily see that $A_{\xi} \cdot J \cdot A_{\xi}=A_{\eta} \cdot J \cdot A_{\eta}=0$. We get the desired result.

COROLLARY 4.3. There exists Einstein, *-Einstein almost Hermitian structure on $S^{3}(r) \times S^{3}(r)$ which is not a quasi-Kähler structure.

PROOF. By Theorem B in [9], the induced almost Hermitian structure on $S^{3}(r) \times S^{3}(r)$ is not quasi-Kähler.

REMARK. The complex structure on $S^{3} \times S^{3}$ which is defined by Calabi-Eckmann is never *-Einstein [13]. Also the 3 -symmetric space $S p(1) \times S p(1) \times S p(1) / S p(1) \simeq S^{3} \times S^{3}$ is a nearlyKälher Einstein, *-Einstein manifold where $S p(1)$ is embedded diagonally in $S p(1) \times S p(1) \times S p(1)$. Hence the induced almost complex structure is different from the above two almost complex structures.

Next, we shall study fundamental relations between *-scalar curvature and the second fundamental form of $M^{6}$.

PROPOSITION 4.4. Let $M^{6}=\left(M^{6}, J,<,>\right)$ be an almost Hermitian submanifold in $\mathbf{O}$. $M^{6}$ is a minimal submanifold in $\mathbf{O}$ and $\tau=\tau *$ if and only if $M^{6}$ is a Hermitian manifold.

PROOF. By (2) \& (4) of Proposition 2.1 and Proposition 3.4, we can easily get the desired result.

PROPOSITION 4.5. Let $M^{6}=\left(M^{6}, J<,>\right)$ be an almost Hermitian submanifold in $\mathbf{O}$. $M^{6}$ is a minimal submanifold in $\mathbf{O}$ and $\tau+\tau *=0$ if and only if the immersion is totally geodesic.

PROOF. From (2) \& (4) of Proposition 2.1, we see that $\operatorname{tr} \Pi^{1,1}=0, A=C=0$. By (4) of Proposition 3.2, $M^{6}$ is a Kähler submanifold in $\mathbb{O}$. Hence we have $A=B=C=0$. Hence we get the conclusion.

Lastly, we shall prove the following.

THEOREM 4.6. Let $M^{6}=\left(M^{6}, J,<,>\right)$ be a 6-dimensional almost Hermitian submanifold in $0 . M^{6}$ is a weakly $*$-Einstein, minimal submanifold in $\mathbb{O}$ which satisfy $\tau=\tau *$, if and only if 
the immersion is totally geodesic.

To prove Theorem 4.6, we prepare threc Lemmas. We define the corresponding 2 -form $\psi$ of the Ricci $*$-tensor as follows:

$$
\psi(x, y)=\frac{\sqrt{-1}}{2} \sum_{i=1}^{6}\left\langle R\left(\epsilon_{2}, J \epsilon_{\imath}\right), y, x\right\rangle
$$

for any $x, y \in T_{p} M^{6}$ Then we have the following

LEMMA 4.7. $\psi=\sqrt{-1}\left({ }^{t} \bar{\eta} \wedge \eta-{ }^{t} \theta \wedge \bar{\theta}\right)$

$$
=\sqrt{-1}\left({ }^{t} \omega(A \bar{B}-B C) \wedge \omega+{ }^{t} \omega\left(A \bar{A}-{ }^{t} \bar{B} B-B^{t} \bar{B}+C \bar{C}\right) \wedge \bar{\omega}-{ }^{t} \bar{\omega}(\bar{A} B-\bar{B} \bar{C}) \wedge \bar{\omega}\right) .
$$

PROOF. By (3) of Proposition 3.1 and (2.9), we get the disired result.

From Lemma 4.7 and (2.4), we have

$$
d \psi=-2 \sqrt{-1}\left\{{ }^{t} \theta \wedge[\theta] \wedge \eta-{ }^{t} \overline{\theta \wedge[\theta] \wedge \eta}\right\} .
$$

LEMMA 4.8. Let $M^{6}=\left(M^{6}, J,<,>\right)$ be a Hermitian, weakly *-Einstein submanifold in $\mathbf{O}$. Then $d \psi=0$.

PROOF. Since $M^{6}$ is a Hermitian submanifold in $\mathbb{O}$, we have

$$
b=\bar{A} \bar{\omega}, \theta=\bar{C} \bar{\omega} .
$$

By (4.1) and (4.2), we have $d \psi \in \Lambda^{3,0} \oplus \Lambda^{0,3}$.

On the other hand, by (1) of Proposition 4.1 and Proposition 4.7, we have

$$
d \psi=-d\left(\frac{\tau *}{6} \Omega\right) \in \Lambda^{1,2} \oplus \Lambda^{2,1}
$$

Hence, we get the desired result.

We recall the following.

LEMMA 4.9 ([1], Prop. 3.7). Let $M^{6}=\left(M^{6}, J,<,>\right)$ be a Hermitian submanifold in $\mathbf{O}$. Then we have $\operatorname{rank} C(p) \leq 1$ and that if $\operatorname{rank} C(p)=1$, then there exists a neighborhood $U$ of $p$ such that $C={ }^{t} c c$ and $A=\frac{1}{2}\left({ }^{t} a c+{ }^{t} c a\right)$, where $a, c$ are $M_{1 \times 3}(\mathbb{C})$-valued functions on $\mathcal{F}(U)$ which are well-defined up to sign.

We are now in a position to prove Theorem 4.6. If $\operatorname{rank} C=1$, by Lemma 4.8 , we have

$$
d(\tau * \Omega)=d \tau * \wedge\left(\frac{\sqrt{-1}}{2}{ }^{t} \omega \wedge \bar{\omega}\right)+\frac{\sqrt{-1}}{2} \tau *\left({ }^{t} \omega \wedge[\omega] \wedge \bar{C} \bar{\omega}-{ }^{t} \bar{\omega} \wedge[\bar{\omega}] \wedge C \omega\right)=0 .
$$

By (4.3) and $\omega^{1} \wedge \omega^{2} \wedge \bar{\omega}^{3}, \omega^{3} \wedge \omega^{1} \wedge \bar{\omega}^{2}, \omega^{2} \wedge \omega^{3} \wedge \bar{\omega}^{1}$ are linearly independent, we have $C_{1}^{1}=C_{2}^{2}=C_{3}^{3}=0$. By Lemma 4.9, we have $C=0$. This is a contradiction. From this argument, we see that $\operatorname{rank} C \equiv 0$. Therefore $M^{6}$ is a Kähler submanifold in $\mathbf{O}$. A *-Einstein Kähler manifold is necessarily an Einstein Kähler manifold. Hence $M^{6}$ is an Einstein Kähler submanifold in $\mathbb{C}^{4} \simeq\left(\mathbb{O}, J_{u}\right)$. By Chern's theorem in [4], we get the desired result.

ACKNOWLEDGEMENT. The author would like to express his hearty thanks to Professor Sekigawa and the referee for their valuable suggestions.

\section{REFERENCES}

1. BRYANT, R.L., Submanifolds and special structures on the octonians, J. Diff. Geom. 17 (1982), 185-232.

2. BRYANT, R.L., Conformal and minimal immersions of compact surfaces into the 4-sphere, J. Diff. Geom. 17 (1982), 455-473. 
3. CALABI, E., Construction and properties of some 6-dnuchsional manifolds, Trans Amer. Math. Soc. 87 (1958), 407-43S.

4. CHERN, S.S., On Einstein hypersurfaces in Kählenian nunifold of constant holomorphnc sectional curvature, J. Diff. Geom 1 (1967), 21-31.

5. GRAY, A., Vector cross products on manifolds, Trans. Amer. Math. Soc. 141 (1969), 465504.

6 GRAY, A. and HERVELLA, L., The sixteen classes of almost Hermitian manifolds, Ann. Math. Pura ed Appl. 123 (1980), 35-58.

7. GUADALUPE, I.V. and RODRIGUEZ, L., Normal curvature of surfaces in spaces forms, Pacific J. of Math. 106 (1983), 95-102.

8. HARVEY, R. and LAWSON, H.B., Jr., Calibrated geometries, Acta. Math. 148 (1982), 47 157.

9. HASHIMOTO, H., Some 6-dimensional oriented submanitold in the octonians, Math. Rep. Toyama Univ. 11 (1988), 1-19.

10. HASHIMOTO, H., Oriented 6-dimensional submanifolds in the octonians 0. Geometry of manifolds (edited by Shiohama), Academic Press, (1989), 71-93.

11. HASHIMOTO, H., Characteristic classes of oriented 6-dimensional submanifolds in the octonians, Kodai Math. J. 16 (1993), 65-73.

12. KODA, T., A remark on the manifold $\mathbb{C P}^{2} \# \overline{\mathbb{C P}}^{2}$ and Berard-Bergery's metric (preprint).

13. TRICERRI, F. and VANHECKE, L., Curvature tensors on almost Hermitian manifolds, Trans. Amer. Soc 267 (1981), 365-398.

14. SEKIGAWA, K. and VANHECKE, L., Four-dimensional almost Kähler Einstein manifolds, Ann. Math. Pura ed Appl. CLVII (1990), 149-160.

15. YANO, K. and SUMITOMO, T., Differential geometry on hypersurfaces in a Cayley space, Proc. Roy Soc. Edin. 66 (1964), 216-231. 


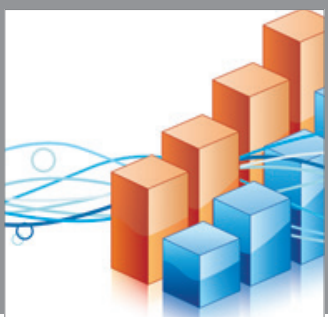

Advances in

Operations Research

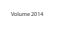

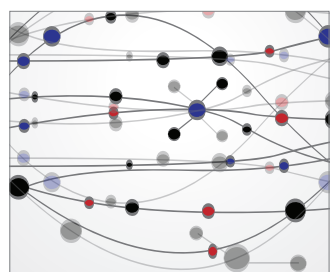

\section{The Scientific} World Journal
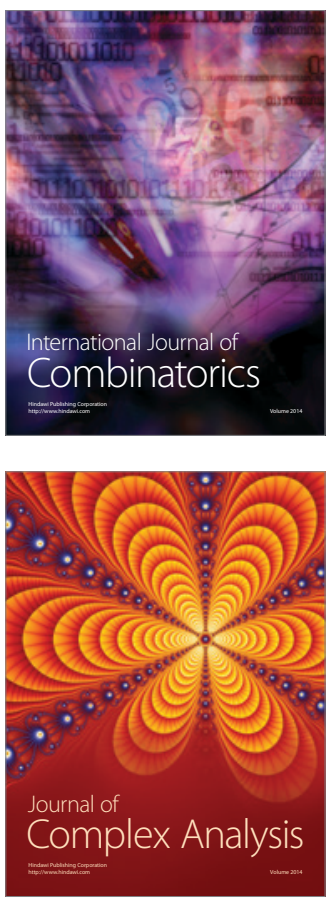

International Journal of

Mathematics and

Mathematical

Sciences
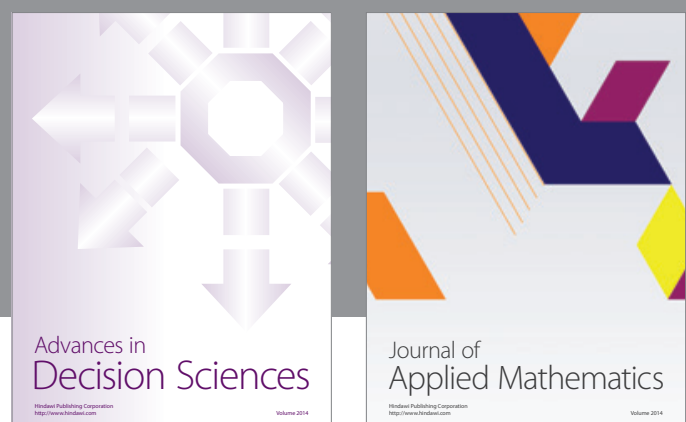

Journal of

Applied Mathematics
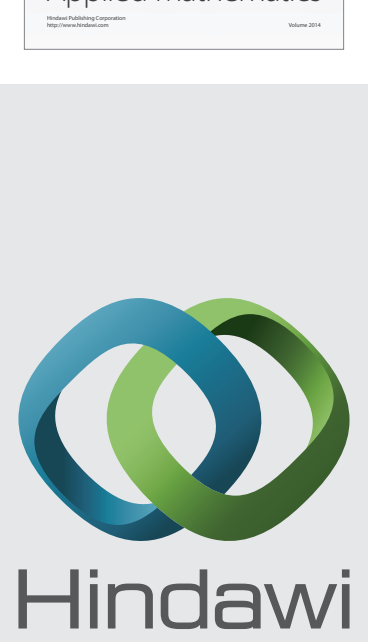

Submit your manuscripts at http://www.hindawi.com
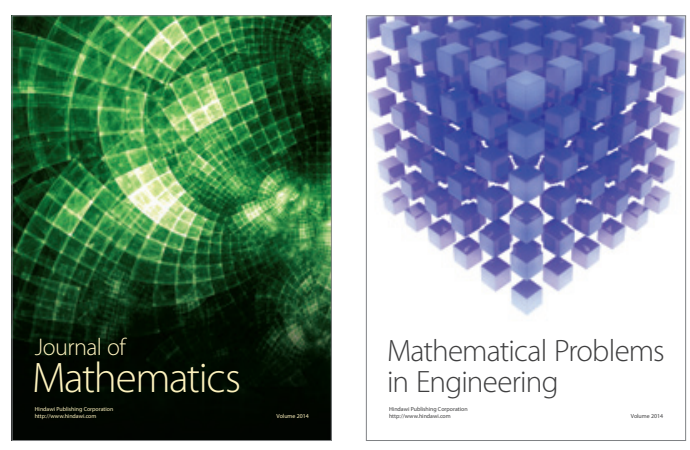

Mathematical Problems in Engineering
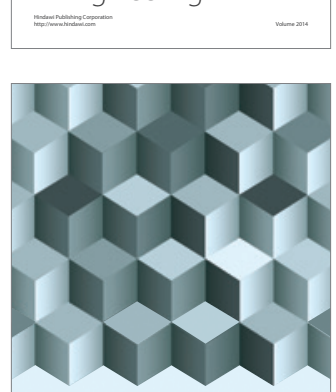

Journal of

Function Spaces
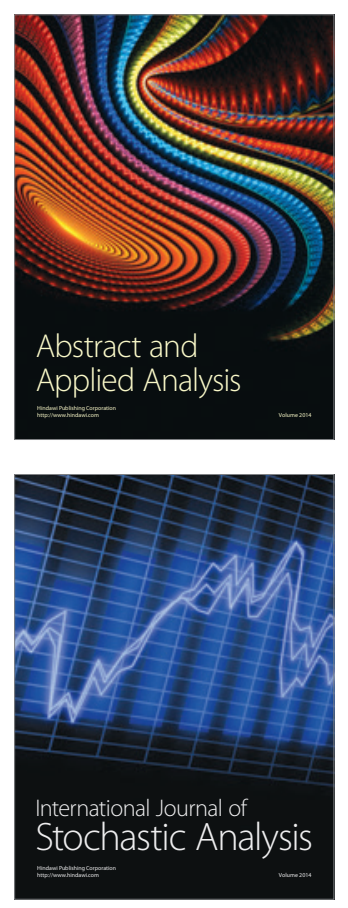

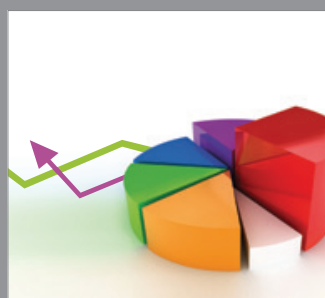

ournal of

Probability and Statistics

Promensencen
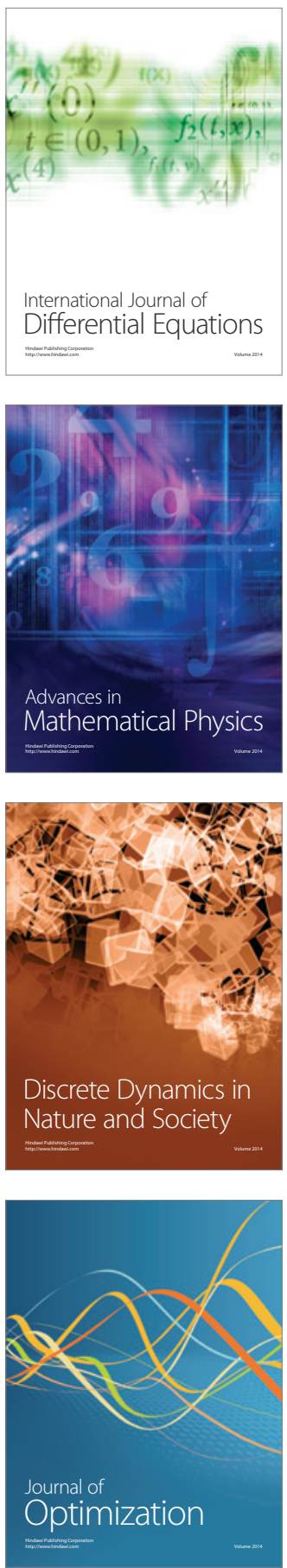